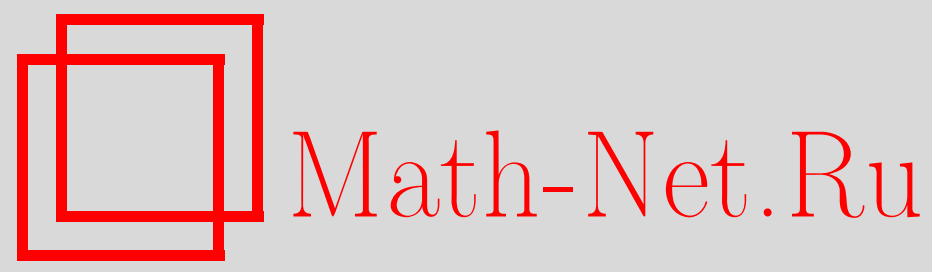

А. С. Тихомиров, Т. Л. Трошина, Бирациональная и численная геометрия многообразия полных пар двоеточий алгебраической поверхности, Матем. заметки, 1999, том 65, выпуск 3, 412-419

DOI: https://doi.org/10.4213/mzm1065

Использование Общероссийского математического портала Math-Net.Ru подразумевает, что вы прочитали и согласны с пользовательским соглашением http://www . mathnet.ru/rus/agreement

Параметры загрузки:

IP : 54.198 .187 .58

26 апреля 2023 г., $16: 36: 03$

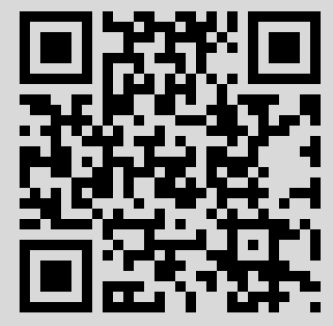




\title{
БИРАЦИОНАЛЬНАЯ И ЧИСЛЕННАЯ ГЕОМЕТРИЯ МНОГООБРАЗИЯ ПОЛНЫХ ПАР ДВОЕТОЧИЙ АЛГЕБРАИЧЕСКОЙ ПОВЕРХНОСТИ
}

\author{
А. С. Тихомиров, Т. Л. Трошина
}

Работа посвящена описанию бирациональных перестроек, необходимых для регуляризации "сложения" пар точек на алгебраической поверхности. Образцом описания служит сложение точек алгебраической поверхности, требующей однократного раздутия гладкого подмногообразия - диагонали прямого произведения поверхности на себя. Точное описание раздутий приводит к описанию геометрии (кольца алгебраических циклов) и численных инвариантов - произведений классов Черна стандартных расслоений на схемах Гильберта порядка 4 алгебраических поверхностей.

Библиограффия: 5 названий.

0. Введение. Пусть $S$ - гладкая, проективная, неприводимая алгебраическая поверхность над полем $k=\bar{k}$, char $k \neq 2, H_{d}=$ Hilb $^{d} S$ - схема Гильберта 0-мерных подсхем длины $d$ ( $d$-точий) на $S, Z$ - подсхема в $S$, соответствующая данной замкнутой точке $z \in H_{d}$. В статье [1] для целых $d_{1}, d_{2} \geqslant 1$ было введено понятие многообразия $X_{d_{1} d_{2}}$ полных пар 0-мерных подсхем длин $d_{1}, d_{2}$ в $S$ как замыкания в $H_{d_{1}} \times H_{d_{2}} \times H_{d_{1}}+d_{2}$ рационального морфизма "сложения точек" $f: H_{d_{1}} \times H_{d_{2}} \rightarrow H_{d_{1}+d_{2}}:\left(z_{1}, z_{2}\right) \mapsto z$, где $Z=Z_{1} \cup Z_{2}$, регулярного на $H_{d_{1}} \times H_{d_{2}} \backslash \Gamma$, где $\Gamma=\Gamma_{d_{1} d_{2}}=\left\{\left(z_{1}, z_{2}\right) \in H_{d_{1}} \times H_{d_{2}} \mid Z_{d_{1}} \cap\right.$ $\left.Z_{d_{2}} \neq \varnothing\right\}-$ график инциденции. Там же доказано, что в случае $d_{1}=d_{2}=2$ многообразие $X=X_{22}$ (называемое ниже многообразием полных пар двоеточий) гладко, и естественная проекция $\sigma: X \rightarrow H_{2} \times H_{2}$ есть раздутие $X$ вдоль $Г$ как приведенного многообразия. Так как $\Gamma=\Gamma_{22}$ - особое многообразие, естественно разложить $\sigma$ в композицию раздутий и стягиваний с неособыми центрами. В настоящей заметке мы даем ответ на этот вопрос (теорема 1 ниже) для случая char $k=0$. Полученньй результат позволяет дать нам эффективное описание кольца Чжоу $A(X)$ многообразия $X$ (как градуированного $\mathbb{Z}$-модуля) в терминах кольца $A\left(H_{2} \times H_{2}\right)$ и колец Чжоу других многообразий, участвующих в описании морфизма $\sigma$ (см. формулы (14)-(16)). Это описание позволяет затем в интересном для приложений случае $S=\mathbb{P}^{2}$ выгислить ранги градуированных компонент $A^{\prime}(X)$ кольца $A(X)$ (как свободных $\mathbb{Z}$-модулей), т.е. (в случае $k=\mathbb{C})$ числа Бетти многообразия $X$ (теорема 2$)$.

1. Два последовательных раздутия $\tilde{X} \rightarrow X_{1} \rightarrow X$ многообразия $X$ полных пар двоеточий поверхности $S$. Для описания морфизма $\sigma: X \rightarrow H \times H$, где $H=H_{2}$, нам потребуются некоторые конструкции и результаты из [1]. Пусть $\Gamma_{1}$ и $\Gamma_{2}-$ прообразы на многообразии $T=H \times S \times H$ графиков инциденции (универсальных циклов) $\Gamma_{21} \subset H \times S$ и $\Gamma_{12} \subset S \times H$ при проекциях $\operatorname{pr}_{12}: T \rightarrow H \times S$ и $_{23}: T \rightarrow S \times H$ соответственно. Тогда по определению $\Gamma=\operatorname{pr}_{13}\left(\Gamma^{\prime}\right)$, где $\Gamma^{\prime}=\Gamma_{1} \cap \Gamma_{2}, \operatorname{apr}_{13}: T \rightarrow H \times H-$ 
проекция. Заметим, что универсальный цикл $\Gamma_{12}-$ гладкое подмногообразие в $S \times H$, изоморфное раздутию $\widehat{S \times S}$ многообразия $S \times S$ вдоль диагонали, поэтому естественная проекция $p_{s}: \Gamma_{12} \rightarrow S$ (соответственно $p_{s}^{\prime}: \Gamma_{21} \simeq \Gamma_{12} \stackrel{p_{s}}{\rightarrow}$ ) является плоским гладким морфизмом, а значит, и морфизм $p_{s}^{\prime} \times p_{s}: \Gamma_{21} \times \Gamma_{12} \rightarrow S \times S$ гладкий. При этом по построению $\Gamma^{\prime} \simeq\left(p_{s}^{\prime} \times p_{s}\right)^{-1}\left(S_{\Delta}\right)$, где $S_{\Delta}$ - диагональ в $S \times S$, причем морфизм $\pi_{s}=p_{s}^{\prime} \times p_{s} \mid \Gamma^{\prime}: \Gamma^{\prime} \rightarrow S_{\Delta} \simeq S$ совпадает с композицией $\Gamma^{\prime} \hookrightarrow T \stackrel{\mathrm{pr}_{2}}{\rightarrow} S$. Следовательно, $\Gamma^{\prime}$ - гладкое многообразие. Далее, пусть $\Delta$ - диагональ в $H \times H, \Delta \simeq H, p=\operatorname{pr}_{13} \mid \Gamma^{\prime}$, $\Delta^{\prime}=p^{-1}(\Delta)=\operatorname{pr}_{13}^{-1}(\Delta) \cap \Gamma^{\prime}$. По построению $\Delta^{\prime}$ изоморфно универсальному циклу $\Gamma_{12} \subset S \times H$ при изоморфизме $\operatorname{pr}_{13}^{-1}(\Delta) \simeq S \times H$, причем пересечение в $T$ гладких многообразий $\operatorname{pr}_{13}^{-1}(\Delta)$ и $\Gamma^{\prime}$ вдоль $\Delta^{\prime}$ как гладкого подмногообразия в $T$ трансверсально. Кроме того, полагая $\Gamma^{*}=\Gamma \backslash \Delta, \Gamma^{*}=\Gamma^{\prime} \backslash \Delta^{\prime}$, мы имеем изоморфизм $p \mid \Gamma^{*}: \Gamma^{\prime *} \rightarrow \Gamma^{*}$ и двойное накрытие $p \mid \Delta^{\prime}: \Delta^{\prime} \rightarrow \Delta$, совпадающее с двойным накрытием $\widetilde{S \times S} \rightarrow H$ при указанных выше отождествлениях. В частности, $\Delta=\operatorname{Sing} \Gamma-$ множество двойных точек Г и $p: \Gamma^{\prime} \rightarrow \Gamma-$ морфизм нормализации.

Пусть $\sigma_{0}: \widetilde{\Gamma} \rightarrow \Gamma$ - раздутие $\Gamma$ с центром в многообразии $\Delta$ и $_{\Gamma_{\Gamma^{\prime}}}: \widetilde{\Gamma}^{\prime}=\widetilde{\Gamma} \times_{\Gamma} \Gamma^{\prime} \rightarrow \Gamma^{\prime}$ - естественная проекция. С другой стороны, пусть $\widehat{\Gamma}$-раздутие $\Gamma^{\prime}$ с центром в гладком многообразии $\Delta^{\prime}$. Ввиду гладкости $\Gamma^{\prime}$ многообразие $\widehat{\Gamma}$ также гладко. Кроме того, поскольку в силу сказанного вьше многообразие $\Delta^{\prime}$ является схемным прообразом $\Delta$ при проекции $p$, по известному функториальному свойству раздутий (см., например, $[2$, дополнение В.6.9]) имеем вложение $\widehat{\Gamma} \stackrel{i}{\hookrightarrow} \widetilde{\Gamma}^{\prime}$, так что $\operatorname{pr}_{\Gamma^{\prime}}$ о $i: \widehat{\Gamma} \rightarrow \Gamma^{\prime}-$ морфизм раздутия с центром $\Delta^{\prime}$.

Рассмотрим теперь индуцированњый морфизм $\widetilde{p}=\operatorname{pr}_{\widetilde{\Gamma}} \circ i: \widehat{\Gamma} \rightarrow \widetilde{\Gamma}$, где $\operatorname{pr}_{\widetilde{\Gamma}}: \widetilde{\Gamma}^{\prime} \rightarrow \widetilde{\Gamma}-$ естественная проекция. Так как согласно сказанному выше $p$ - конечный морфизм степени 1 в общей точке, то и $\widetilde{p}$ - также конечньй морфизм неприводимых многообразий степени 1 в общей точке.

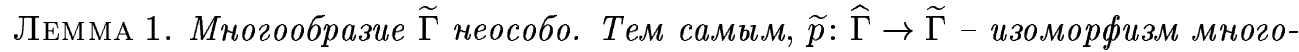
образий.

ДоКАЗАТЕЛЬСТво. Пусть $\sigma_{\Delta}: X_{1} \rightarrow H \times H-$ раздутие с центром в многообразии $\Delta \subset H \times H$. Имеем коммутативную диаграмму

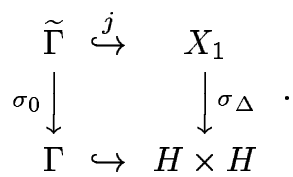

Для описания $\widetilde{\Gamma}$ воспользуемся локальными уравнениями подмногообразия $\Gamma$ в $H \times H$, полученньпи в $\S 4$ работы [1]. Именно, достаточно рассмотреть эти уравнения в подходящей окрестности $U$ точки $x_{0}=(z, z) \in \Delta \subset H \times H$, где $Z$ - неприведенная схема длины 2. Согласно [1, формулы (4.16)-(4.21) и (4.26)] можно выбрать окрестность $U$ с локальными координатами $a, b, c, d, a_{2}, b_{2}, c_{2}, d_{2}$ такими, что идеалы $I_{\Gamma}=I_{\Gamma \cap U, U}$ и $I_{\Delta}=T_{\Delta \cap U, U}$ суть

$$
\begin{gathered}
I_{\Gamma}=\left(b^{2}+4 a^{2} b_{2}, 2 a d-b c, d^{2}+c^{2} b_{2}, b d+2 a c b_{2}\right), \\
I_{\Delta}=(a, b, c, d) .
\end{gathered}
$$


Пользуясь (2), покроем $\sigma_{\Delta}^{-1}(U)$ картами $U_{i}, i=1,2,3,4$, с координатами $x_{i j}, j=1, \ldots, 8$, в $U_{i}$ такими, в которых $\sigma_{i}=\sigma_{\Delta} \mid U_{i}: U_{i} \rightarrow U$ задается формулами

$$
\begin{gathered}
\sigma_{1}: a=x_{11}, \quad b=x_{21} x_{11}, \quad c=x_{31} x_{11}, \quad d=x_{41} x_{11}, \\
x_{51}=a_{2}, \quad x_{61}=b_{2}, \quad x_{71}=c_{2}, \quad x_{81}=d_{2}, \\
\sigma_{2}: a=x_{12} x_{22}, \quad b=x_{22}, \quad c=x_{32} x_{22}, \quad d=x_{42} x_{22}, \\
x_{52}=a_{2}, \quad x_{62}=b_{2}, \quad x_{72}=c_{2}, \quad x_{82}=d_{2}, \\
\sigma_{3}: a=x_{13} x_{33}, \quad b=x_{23} x_{33}, \quad c=x_{33}, \quad d=x_{43} x_{33}, \\
x_{53}=a_{2}, \quad x_{63}=b_{2}, \quad x_{73}=c_{2}, \quad x_{83}=d_{2}, \\
\sigma_{4}: \quad a=x_{14} x_{44}, \quad b=x_{24} x_{44}, \quad c=x_{34} x_{44}, \quad d=x_{44}, \\
x_{54}=a_{2}, \quad x_{64}=b_{2}, \quad x_{74}=c_{2}, x_{84}=d_{2} .
\end{gathered}
$$

Пусть $\widetilde{\Delta}=\sigma_{\Delta}^{-1}(\Delta), \widetilde{\Delta}_{i}=\widetilde{\Delta} \cap U_{i}, I_{\widetilde{\Delta}_{i}}=I_{\widetilde{\Delta}_{i}, U_{i}}, i=1,2,3,4$. Из (2) и (3) немедленно следует, что

$$
I_{\widetilde{\Delta}_{i}}=\left(x_{i i}\right), \quad i=1,2,3,4 .
$$

Подставляя (3) в (1), с помощью элементарных вычислений получаем (после удаления из $U_{i} \backslash \sigma_{\Delta}^{-1}\left(x_{0}\right)$ подходяших замкнутых подмножеств) формулы для идеалов $I_{\sigma_{i}^{-1}}(\Gamma \cap U), U_{i}$ $=\sigma_{i}^{-1} I_{\Gamma}$ :

$$
\begin{gathered}
\sigma_{1}^{-1} I_{\Gamma}=\left(x_{11}\right)^{2}\left(4 x_{61}+x_{21}^{2}, 2 x_{41}-x_{21} x_{31}\right), \quad \sigma_{2}^{-1} I_{\Gamma}=\left(x_{22}\right)^{2}, \\
\sigma_{3}^{-1} I_{\Gamma}=\left(x_{33}\right)^{2}\left(2 x_{13} x_{43}-x_{23}, x_{43}^{2}+x_{63}\right), \quad \sigma_{4}^{-1} I_{\Gamma}=\left(x_{44}\right)^{2} .
\end{gathered}
$$

Как следует из $(5), \sigma_{i}^{-1} I_{\Gamma}$ делится на $I_{\widetilde{\Delta}_{i}}^{2}, i=1,2,3,4$. Сокращая эти формулы на обратимые идеалы $I_{\widetilde{\Delta}_{i}}^{2}$, получаем формулы для искомых идеалов $I_{\widetilde{\Gamma}_{i}}=I_{\widetilde{\Gamma}_{i}, U_{i}}$, где $\widetilde{\Gamma}_{i}=\widetilde{\Gamma} \cap U_{i}$, $i=1,2,3,4$ :

$I_{\widetilde{\Gamma}_{1}}=\left(4 x_{61}+x_{21}^{2}, 2 x_{41}-x_{21} x_{31}\right), \quad I_{\widetilde{\Gamma}_{3}}=\left(x_{23}-2 x_{13} x_{43}, x_{63}+x_{43}^{2}\right), \quad I_{\widetilde{\Gamma}_{2}}=I_{\widetilde{\Gamma}_{4}}=(1)$.

Из формул (5) очевидньм образом следует гладкость $\widetilde{\Gamma}$. Наконец, поскольку $\widetilde{p}: \widehat{\Gamma} \rightarrow \widetilde{\Gamma}-$ конечньй бирациональный морфизм гладких проективных многообразий, то $\widetilde{p}$ - изоморфизм (см., например, [3, гл. II, $\S 4$, следствие 1 из теоремы 2]).

Пусть теперь $\sigma_{\widetilde{\Gamma}}: \widetilde{X} \rightarrow X_{1}$ - раздутие с центром в гладком многообразии $\widetilde{\Gamma}, E=\sigma_{\widetilde{\Gamma}}^{-1}(\widetilde{\Gamma})$ - исключительный дивизор раздутия $\sigma_{\widetilde{\Gamma}}, D=\sigma_{\widetilde{\Gamma}}^{-1}(\widetilde{\Delta}), g_{E}=\sigma_{\widetilde{\Gamma}} \mid E: E \rightarrow \widetilde{\Gamma}$ и $g_{D}=\sigma_{\widetilde{\Gamma}} \mid D:$ $D \rightarrow \widetilde{\Delta}$ - проекции. Из (4)-(6) непосредственно следуют соотношения между пучками идеалов $\mathscr{I}_{\Gamma, H \times H}, \mathscr{I}_{\widetilde{\Delta}, X_{1}}$ и $\mathscr{I}_{\widetilde{\Gamma}, X_{1}}$ :

$$
\sigma_{\Delta}^{-1} \mathscr{I}_{\Gamma, H \times H} \simeq \mathscr{I}_{\widetilde{\Delta}, X_{1}}^{2} \mathscr{I}_{\widetilde{\Gamma}, X_{1}}=\mathscr{I}_{\widetilde{\Gamma}, X_{1}}(-2 \widetilde{\Delta}),
$$

откуда

$$
\left(\sigma_{\Delta} \sigma_{\widetilde{\Gamma}}\right)^{-1} \mathscr{I}_{\Gamma, H \times H}=\mathscr{I}_{D, \tilde{X}}^{2} \mathscr{I}_{E, \tilde{X}}=\mathscr{O}_{\widetilde{X}}(-2 D-E) .
$$

Кроме того, уравнения (4) и (6) немедленно влекут гладкость многообразия $\widetilde{\Delta} \cap \widetilde{\Gamma}, \mathrm{a}$ поскольку $\widetilde{\Delta}-$ гладкий дивизор в $X_{1}$, то отсюда опять по функториальности раздутий получаем, что $g_{D}: D \rightarrow \widetilde{\Delta}-$ раздутие с гладким центром $\widetilde{\Delta} \cap \widetilde{\Gamma}$, откуда следует гладкость $D$. 
Далее, по определению $E \simeq \mathbb{P}\left(\mathscr{N}_{\widetilde{\Gamma} / X_{1}}^{\vee}\right)$ и $g_{E}$ совпадает со структурным морфизмом $\mathbb{P}\left(\mathcal{N}_{\widetilde{\Gamma} / X_{1}}^{\vee}\right) \rightarrow \widetilde{\Gamma}$, а значит, слой $g_{E}$ изоморфен $\mathbb{P}^{1}$. Аналогично, пусть $g_{\Delta}: \widetilde{\Delta} \simeq \mathbb{P}\left(\mathcal{N}_{\Delta / H \times H}^{\vee}\right)$ $\simeq \mathbb{P}\left(\Omega_{H}\right) \rightarrow H \simeq \Delta-$ структурный морфизм и пусть $\mathbb{P}_{z}^{3}=g_{\Delta}^{-1}(z), E_{z}=E \cap \mathbb{P}_{z}^{3}$, $z \in \Delta \simeq H$. Элементарное наблюдение показывает, что если соответствующая точке $z$ схема $Z$ в $S$ приведена (т.е. $Z$ - пара различных точек в $S$ ), то схема $E_{z}$ есть объединение двух скрещивающихся прямых $l_{1 z} \cup l_{2 z}$ в $\mathbb{P}_{z}^{3}$ (рассматриваемых как неприводимые многообразия). Соответственно из формул (6) непосредственно следует, что если $Z$-неприведенная схема $\left(Z \simeq \operatorname{Spec} k[x] /\left(x^{2}\right)\right)$, то носитель схемы $E_{z}$ есть некоторая прямая $l_{z}$ в $\mathbb{P}_{z}^{3}$, а схемная структура $E_{z}$ совпадает с двойной структурой на $l_{z}$ как образующей некоторой гладкой квадрики $V_{z} \subset \mathbb{P}_{z}^{3}: \mathscr{O}_{E_{z}}=\mathscr{O}_{V_{z}} / \mathscr{O}_{V_{z}}\left(-2 l_{z}\right)$ (здесь квадрика $V_{z}$ может быть выбрана неоднозначно).

Далее, пусть $G(1,3)_{z}$ - грассманиан прямых пространства $\mathbb{P}_{z}^{3}$ и $Q_{z}=\left\{l \in G(1,3)_{z} \mid\right.$ $\left.\chi\left(\mathscr{O}_{l \cap E_{z}}\right)=2\right\}$. Кроме того, пусть $B_{H}=\{z \in H \mid Z$ неприведена $\}$ и $H^{*}=H \backslash B_{H}$. Из предыдущего описания схемы $E_{z}$ непосредственно вытекает

ЛЕмма 2. Если $z \in H^{*}$, то $Q_{z} \simeq l_{1 z} \times l_{2 z}-$ гладкая квадрика. Если $z \in B_{H}$, то

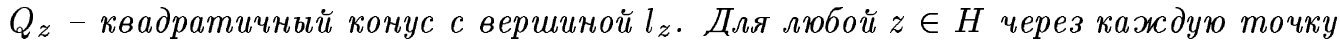
в $\mathbb{P}_{z}^{3} \backslash E_{z}$ проходит единственная прямая из $Q_{z}$.

Рассмотрим теперь множество $Y^{\prime}=\bigcup_{z \in \Delta} Q_{z}$ (ниже мы увидим, что на $Y^{\prime}$ существует структура гладкого шестимерного многообразия). Для произвольной прямой $\tilde{l} \in Y^{\prime}$ из свойств раздутия $\sigma_{\Delta}$ немедленно следует, что $(\widetilde{\Delta} \cdot \tilde{l})_{X_{1}}=-1$. Далее, пусть $l-$ собственньй прообраз прямой $\tilde{l}$ при отображении $\sigma_{\widetilde{\Gamma}}$ (или, что то же самое, при морфизме $g_{D}$, поскольку $\tilde{l} \subset \widetilde{\Delta})$. Очевидно, что $\sigma_{l}=\sigma_{\widetilde{\Gamma}} \mid l: l \rightarrow \tilde{l}$-изоморфизм, так что

$$
(l \cdot D)_{\tilde{X}}=\operatorname{deg}\left(\mathscr{O}_{\tilde{X}}(D) \mid l\right)=\operatorname{deg} \sigma_{l}^{*}\left(\mathscr{O}_{X_{1}}(\widetilde{\Delta}) \mid \tilde{l}\right)=\operatorname{deg}\left(\mathscr{O}_{X_{1}}(\widetilde{\Delta}) \mid \tilde{l}\right)=(\widetilde{\Delta} \cdot \tilde{l})_{X_{1}}=-1 .
$$

Кроме того, пусть $\tilde{l} \in Q_{z}$, где $z \in \Delta^{*}$. Тогда по определению $\tilde{l}$ пересекает $\widetilde{\Gamma}$ трансверсально в двух различных точках и, как следствие, $(l \cdot E)_{\tilde{X}}=2$. Отсюда и из $(8)$ получаем $((E+2 D) \cdot l)_{\tilde{X}}=0$. Это равенство будет использовано в следующем пункте.

В заключение этого пункта заметим, что в силу леммы 2 прямые $l=\sigma_{l}^{-1}(\tilde{l}), \tilde{l} \in Y^{\prime}$, заметают дивизор $D$ на $\widetilde{X}$, и через каждую точку $D$ проходит единственная такая прямая $l$. Тем самьм, определено отображение множеств $g: D \rightarrow Y^{\prime}: x \mapsto \sigma_{l}(l)$, где $l$ определена однозначно условием $x \in l$. Ниже мы покажем, что в действительности $g: D \rightarrow Y^{\prime}$ является морфизмом, и дадим его точное описание (теорема 1 ).

2. Разложение морфизма $\sigma: X \rightarrow H \times H$ в “домик” Хиронаки. Целью этого пункта является доказательство существования бирационального регулярного морфизма $\widetilde{\sigma}: \widetilde{X} \rightarrow X$ и его точное описание, приводящее затем к основному результату о разложении $\sigma: X \rightarrow H \times H$ в “домик” Хиронаки (теорема 1). Для этого нам потребуется провести несколько дополнительных построений. Пусть $\Delta^{*}-$ образ $H^{*}$ при изоморфизме $\Delta \simeq H$. Сначала возьмем произвольную точку $z \in \Delta^{*}$ и пусть $Z=x_{1} \cup x_{2}-$ соответствующая ей пара различных точек в $S$. Выбирая достаточно малую аналитическую окрестность $U \subset H \times H$ точки $z$ (здесь для удобства мы временно ограничиваемся случаем $k=\mathbb{C}$ ), непосредственно замечаем (пользуясь, например, формулами (1)), что $\Gamma \cap U$ является объединением двух своих гладких ветвей $\Gamma_{(1)}$ и $\Gamma_{(2)}$, трансверсально пересекающихся вдоль $\Delta \cap U$. Тем самьм, полагая $\widetilde{U}=\sigma^{-1}(U)$, мы получаем, что раздутие $\sigma_{U}=\sigma \mid \widetilde{U}: \widetilde{U} \rightarrow U$ (в категории аналитических пространств) разлагается в 
композицию $\widetilde{U} \stackrel{\sigma_{2}}{\rightarrow} U^{\prime} \stackrel{\sigma_{1}}{\rightarrow} U$, где $\sigma_{1}: U^{\prime} \rightarrow U-$ раздутие с центром в $\Gamma_{(1)}$, а $\sigma_{2}: \widetilde{U} \rightarrow U^{\prime}-$ раздутие с центром в $\Gamma_{(2)}^{\prime}=\sigma_{1}^{-1}\left(\Gamma_{(2)}\right)$. При этом $\delta_{2}=\sigma_{1} \mid \Gamma_{(2)}^{\prime}: \Gamma_{(2)}^{\prime} \rightarrow \Gamma_{(2)}-$ раздутие с центром $\Delta \cap U$ в $\Gamma_{(2)}$, так что, полагая $E_{i}=\sigma^{-1}\left(\Gamma_{(i)}\right), \Gamma_{(i)}^{*}=\Gamma_{(i)} \cap \Gamma^{*}, E_{i}^{*}=\sigma^{-1}\left(\Gamma_{(i)}^{*}\right)$, $i=1,2$, получаем морфизм $\sigma_{E_{2}}=\sigma_{2} \mid E_{2}: E_{2} \rightarrow \Gamma_{(2)}^{\prime}$ такой, что $\sigma_{E_{2}}\left|E_{2}^{*}=\sigma\right| E_{2}^{*}$. Meняя порядок раздутия $U$ вдоль ветвей $\Gamma_{(1)}$ и $\Gamma_{(2)}$, получаем морфизмы $\sigma_{E_{1}}: E_{1} \rightarrow \Gamma_{(1)}^{\prime}$ и $\delta_{1}: \Gamma_{(1)}^{\prime} \rightarrow \Gamma_{(1)}$, где $\delta_{1}-$ раздутие $\Gamma_{(1)}$ с центром $\Delta \cap U$, такие, что $\sigma_{E_{1}}\left|E_{1}^{*}=\sigma\right| E_{1}^{*}$. Пусть $Q(z)=\sigma^{-1}(z)$ и $n_{i z}=\delta_{i}^{-1}(z), i=1,2$; в частности, $n_{2 z}=\delta_{2}^{-1}(z)=\sigma_{1}^{-1}(z)$, $Q(z)=\sigma_{2}^{-1}\left(n_{2 z}\right)$ и по конструкции $Q(z) \simeq n_{1 z} \times n_{2 z} \simeq n_{i z} \times_{\Gamma_{(i)}^{\prime}} E_{i}, i=1,2$ (здесь расслоенные квадраты определяются данными $\left.n_{i z} \hookrightarrow \Gamma_{(i)}^{\prime} \stackrel{\sigma_{E_{i}}}{\leftarrow} E_{i}\right)$, причем проекции на сомножители $Q(z) \stackrel{\mathrm{pr}_{i}}{\rightarrow} n_{i z}$ совпадают с естественными проекциями в расслоенных квадратах. Отсюда и из равенств $\sigma_{E_{i}}\left|E_{i}^{*}=\sigma\right| E_{i}^{*}, i=1,2$, следует

Лемма 3. Прямые $h$ любой серии на квадрике $Q(z)$, әде $z \in \Delta^{*}$, и прямые $\sigma^{-1}(x)$, $x \in \Gamma^{*}$, принадлежат одному алгебраическому семейству прямых на дивизоре $E_{\Gamma}=\sigma^{-1}(\Gamma)$ в $X$. Тем самым,$\left(h \cdot E_{\Gamma}\right)_{X}=-1$.

Вернемся к композиции раздутий $\bar{\sigma}=\sigma_{\Delta} \circ \sigma_{\widetilde{\Gamma}}: \widetilde{X} \rightarrow H \times H$. Так как согласно (7) $\bar{\sigma}^{-1} \mathscr{I}_{\Gamma, H \times H}-$ обратимый пучок, то по универсальности раздутия $\sigma: X \rightarrow H \times H$ получаем, что существует морфизм $\widetilde{\sigma}: \widetilde{X} \rightarrow X$ такой, что $\bar{\sigma}=\sigma \circ \widetilde{\sigma}$; в частности, $\bar{\sigma}^{-1} \mathscr{I}_{\Gamma, H \times H}=\widetilde{\sigma}^{-1} \mathscr{I}_{E_{\Gamma}, X}=\widetilde{\sigma}^{*} \mathscr{O}_{X}\left(-E_{\Gamma}\right)$, где $E_{\Gamma}=\sigma^{-1}(\Gamma)$ - исключительньй дивизор раздутия $\sigma$, а правое равенство здесь следует из обратимости на $\widetilde{X}$ пучков $\widetilde{\sigma}^{-1} \mathscr{I}_{E_{\Gamma}, X}$ и $\widetilde{\sigma}^{*} \mathscr{O}_{X}\left(-E_{\Gamma}\right)$. Более того, равенство (7) дает

$$
\sigma^{*} \mathscr{O}_{X}\left(-E_{\Gamma}\right)=\mathscr{O}_{\tilde{X}}(-E-2 D) .
$$

Заметим теперь, что по определению отображения $\widetilde{\sigma}$, обозначая $Y=\sigma^{-1}(\Delta)$, имеем в силу гладкости $\Gamma^{*}$ изоморфизм $\widetilde{\sigma} \mid \widetilde{X} \backslash D: \widetilde{X} \backslash D \stackrel{\sim}{\rightarrow} X \backslash Y$, откуда $Y=\widetilde{\sigma}(D)$.

Далее, возьмем произвольную пару $(z, y)$, где $z \in \Delta, y \in Q_{z}$, и пусть $l=l_{z, y}=g^{-1}(y)-$ прямая в $D$, соответствующая точке $y$. Так как $\widetilde{\sigma}(D)=Y$, то $m=\widetilde{\sigma}(l) \subset \sigma^{-1}(z)=Q(z)$. Покажем, что $m$ - точка. Рассмотрим сначала случай $z \in \Delta^{*}$, т.е. когда $Q(z)$ - гладкая квадрика, и пусть $h_{1}$ и $h_{2}$ - образующие в Ріс $Q(z)$. Предположим теперь, что $m-$ кривая, так что $m \sim a_{1} h_{1}+a_{2} h_{2}$ для некоторых целых $a_{1}, a_{2} \geqslant 0, a_{1}+a_{2}>0$. Пусть $d-$ степень отображения $\widetilde{\sigma}_{l}=\widetilde{\sigma} \mid l: l \rightarrow m$. Тогда согласно лемме $3 \operatorname{deg}\left(\mathscr{O}_{X}\left(E_{\Gamma}\right) \mid m\right)=-\left(a_{1}+a_{2}\right)$, так что в силу $(9)$ получаем $((E+2 D) \cdot l)_{\tilde{X}}=\operatorname{deg}\left(\sigma^{*} \mathscr{O}_{X}\left(E_{\Gamma}\right) \mid l\right)=-d\left(a_{1}+a_{2}\right)<0$ вопреки (8). Следовательно, $m$ - точка. Теперь положим $B_{\Delta}=\Delta \backslash \Delta^{*} \simeq B_{H}, Y^{*}=\sigma^{-1}\left(\Delta^{*}\right)$, $B_{Y}=Y \backslash Y^{*}=\sigma^{-1}\left(B_{\Delta}\right)$ и заметим, что прямые $l_{z, y}$ для $z \in B_{\Delta}$ лежат в замкнутом подмножестве $\widetilde{\sigma}^{-1}\left(B_{Y}\right)$ в $D$ и, тем самьм, составляют собственное замкнутое подмножество семейства $Y^{\prime}$ прямых $l_{z, y}, z \in \Delta$, на $D$ (здесь семейство $Y^{\prime}$ естественньм образом снабжается алгебраической структурой как редуцированная компонента схемы Гильберта Hilb $D$; при этом $g: D \rightarrow Y^{\prime}$, очевидно, является морфизмом, откуда в силу неприводимости $D$ следует неприводимость $\left.Y^{\prime}\right)$. Отсюда и из неприводимости $Y^{\prime}$ следует по непрерывности, что $m=\widetilde{\sigma}\left(l_{z, y}\right)$ - точка и для любой пары $(z, y), z \in B_{\Delta}$. Таким образом, морфизм $g_{D}=\widetilde{\sigma} \mid D: D \rightarrow Y$ пропускается через $g$, т.е. сушествует отображение $\psi: Y^{\prime} \rightarrow Y$ (априори $\psi$ - не обязательно морфизм) такое, что $g_{D}=\psi \circ g$.

Покажем, что $\psi$ - изоморфизм. В самом деле, $g$ по определению является проективным морфизмом, слои которого суть приведенные проективные прямые, а значит, $g_{*} \mathscr{O}_{D}=\mathscr{O}_{Y^{\prime}}$. Тем самым, поскольку $g_{D}=\psi \circ g-$ морфизм, имеем морфизм пучков 
$g_{d}^{\sharp}: \mathscr{O}_{Y} \rightarrow g_{D *} \mathscr{O}_{D}$, где $g_{D *} \mathscr{O}_{D}=\psi_{*} g_{*} \mathscr{O}_{D}=\psi_{*} \mathscr{O}_{Y^{\prime}}$, т.е. $g_{D}^{\sharp}$ индуцирует морфизм пучков $\psi^{\sharp}: \mathscr{O}_{Y} \rightarrow \psi_{*} \mathscr{O}_{Y^{\prime}}$. Пара $\left(\psi, \psi^{\sharp}\right)$ и определяет $\psi$ как морфизм $Y^{\prime} \rightarrow Y$.

Далее, из леммы 3 в силу того, что $\widetilde{\sigma} \mid \widetilde{X} \backslash D: \tilde{X} \backslash D \rightarrow X \backslash Y$-изоморфизм, непосредственно следует, что образующие прямые на любой квадрике $Q(z) \subset Y^{\prime}, z \in \Delta$, при морфизме $\psi$ изоморфно отображаются на образующие прямые квадрики $Q_{z}$. Отсюда, очевидно, следует, что $\psi: Q(z) \rightarrow Q_{z}$ - изоморфизм для любого $z \in \Delta$ независимо от того, является ли $Q_{z}$ гладкой квадрикой или конусом. Тем самым, $\psi$ - биекция.

Наконец, поскольку $g: D \rightarrow Y^{\prime}$ - морфизм со слоем $\mathbb{P}^{1}$ (тем самьм, $g$ - гладкий мор(ризм) и $D$-гладкое многообразие, то $Y^{\prime}$ - также гладкое многообразие, а значит, ввиду гладкости $Y$ биекция $\psi: Y^{\prime} \rightarrow Y-$ изоморфизм.

Теперь заметим, что согласно Мойшезону [4], так как слой $l$ проекции $g$ удовлетворяет условию (8), определено стягивание $\sigma_{Y}: \widetilde{X} \rightarrow X^{\prime}$ дивизора $D$, т.е. определены гладкое (аналитическое) многообразие $X^{\prime}$ и вложение в него гладкого подмногообразия $Y^{\prime} \hookrightarrow X^{\prime}$ такие, что коммутативна диаграмма

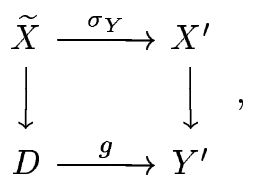

в которой $\sigma_{Y}$ - раздутие $X^{\prime}$ с центром $Y^{\prime}$. Поскольку $g=\sigma_{Y}|D=\tilde{\sigma}| D$, то $\tilde{\sigma}$ как отображение пропускается через $\sigma_{Y}$, т.е. существует отображение $\nu: X^{\prime} \rightarrow X$ такое, что $\tilde{\sigma}=\nu \circ \sigma_{Y}$. Поскольку $\sigma_{Y}$ - раздутие с гладким центром, то, как известно, $\sigma_{Y *} \mathscr{O}_{\tilde{X}}=\mathscr{O}_{X^{\prime}}$, поэтому рассуждение, аналогичное приведенному вьше для морфизма $\psi$, показьвает, что $\nu$ - морфизм (в аналитической категории). Так как по построению $\nu \mid X^{\prime} \backslash Y^{\prime}: X^{\prime} \backslash Y^{\prime} \rightarrow X \backslash Y$ - изоморфизм и $\nu \mid Y^{\prime}=\psi$ - изоморфизм, то $\nu: X^{\prime} \rightarrow X-$ биективный морфизм гладких многообразий, а тем самым, изоморфизм. Следовательно, $\widetilde{\sigma}$ отождествляется с $\sigma_{Y}$; в частности, $\sigma_{Y}$ - раздутие в категории схем над $\mathbb{C}$. Итак, для случая $k=\mathbb{C}$ (а тем самым, по принципу Лефшеца [5, с. 187] и для любого основного поля $k$ характеристики 0$)$ доказана

Tеорема 1. (i) Морфизм раздутия $\sigma: X \rightarrow H \times H$ с иентром Г разлагается $в$ композицию раздутий с гладкими иентрами в диаграмме ( “домике” Хиронаки):

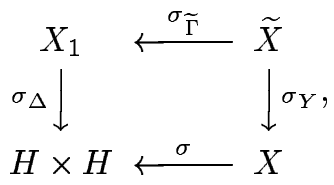

где $\sigma_{\Delta}-$ раздутие $H \times H$ с иентром в диагонали $\Delta \simeq H, \Delta_{\widetilde{\Gamma}}-$ раздутие с гладким иентром $\widetilde{\Gamma}-$ собственным прообразом Г при морфизме $\sigma_{\Delta}$, а $\sigma_{Y}$ - раздутие $c$ гладким иентром $Y=\sigma^{-1}(\Delta)$.

(ii) $\widetilde{\Delta}=\sigma_{\Delta}^{-1}(\Delta) \simeq \mathbb{P}\left(\mathscr{N}_{\Delta / H \times H}^{\vee}\right)$ и $\sigma_{\Delta} \mid \widetilde{\Delta}$ совпадает со структурным морфизмом $g_{\Delta}: \mathbb{P}\left(\mathcal{N}_{\Delta / H \times H}^{\vee}\right) \rightarrow \Delta$.

(iii) $\widetilde{\Gamma}$ изоморфно раздутию многообразия $(\widetilde{S \times S}) \times_{S}(\widetilde{S \times S})$ вдоль гладкого подмногообразия $S \times S$, вложсенного в $(\widetilde{S \times S}) \times{ }_{S}(\widetilde{S \times S})$ как относительная (над $S$ ) 
диагональ. При этом слой естественной проекции $\widetilde{\Gamma} \stackrel{\gamma}{\rightarrow} S$ над произвольной точкой $x \in S$ есть $\widetilde{S_{x} \times S_{x}}-$ раздутие $S_{x} \times S_{x}$ вдоль диагонали, где $S_{x}$ - раздутие $S$ в точке $x$.

Далее, $g_{\Gamma}=\sigma_{\widetilde{\Gamma}} \mid E: E \simeq \mathbb{P}\left(\mathscr{N}_{\widetilde{\Gamma} / X_{1}}^{\vee}\right) \rightarrow \widetilde{\Gamma}$ совпадает со структурным морфизмом проективизачии, где $E$ - исключительный дивизор раздутия $\sigma_{\Gamma}$.

(iv) $g_{Y}=\sigma \mid Y: Y \rightarrow \Delta-$ морфизм со слоем гладкая квадрика над точкой $z \in \Delta^{*}$, соответственно со слоем неприводимый квадратичный конус над точкой $z \in B_{\Delta}$.

(v) Исключительный дивизор $D=\mathbb{P}\left(\mathcal{N}_{Y / X}^{\vee}\right)$ раздутия $\sigma_{Y}: \widetilde{X} \rightarrow X$ с иентром $Y$ совпадает $с \sigma_{\widetilde{\Gamma}}^{-1}(\widetilde{\Delta})$, и $g_{D}=\sigma_{Y} \mid D: D \rightarrow Y-$ структурный морфизм проективизаuиu.

Выше доказаны все утверждения теоремы кроме (iіi). Последнее легко вытекает из предыдущих конструкций.

3. Численная геометрия многообразия $X$. Для проведения дальнейших вычислений сделаем два общих замечания.

ЗАмечаниЕ 1 . Пусть $V$ - произвольное многообразие, $A_{*}(V)=\bigoplus_{k=0}^{\operatorname{dim} V} A_{k}(V)-$ его кольцо Чжоу, градуированное по размерности циклов, $\mathscr{E}$ - локально свободньй пучок ранга $r+1$ на $V, W=\mathbb{P}(\mathscr{E}), \pi: W \rightarrow V$ - структурный морфизм, $\mathscr{O}_{\mathbb{P}(\mathscr{E})}(1)-$ пучок Гротендика, где $\pi_{*} \mathscr{O}_{\mathbb{P}(\mathscr{E})}(1)=\mathscr{E}$, и $\xi_{\pi}=c_{1}\left(\mathscr{O}_{\mathbb{P}(\mathscr{E})}(1)\right)$. Как известно, имеет место изоморфизм градуированных групп

$$
A_{*}(W) \simeq \bigoplus_{i=0}^{r} \pi^{*} A_{*}(V) \cdot \xi_{\pi}^{i} \simeq A_{*}(W / V) \oplus A_{*}(V),
$$

где учтено, что $\pi^{*} A_{*}(V) \cdot \xi_{\pi}^{r} \simeq A_{*}(V)$, и введено обозначение

$$
A_{*}(W / V)=\bigoplus_{i=0}^{r-1} \pi^{*} A_{*}(V) \cdot \xi_{\pi}^{i}=\operatorname{ker}\left(\pi_{*}: A_{*}(W) \rightarrow A_{*}(V)\right) .
$$

В частности, при $r=1$ имеем изоморфизм градуированных групा

$$
A_{*}(W) \simeq \pi^{*} A_{*}(V) \oplus A_{*}(V), \quad A(W / V) \simeq \pi^{*} A_{*}(V) .
$$

ЗАмечАниЕ 2 . Пусть $\mathscr{Z} \hookrightarrow \mathscr{X}$ - регулярное вложение коразмерности $r+1$ многообразия $\mathscr{Z}$ в многообразие $\mathscr{X}, f: \widetilde{\mathscr{X}} \rightarrow \mathscr{X}$ - раздутие с центром $\mathscr{Z}, \mathscr{D}=\mathbb{P}\left(\mathscr{N}_{\mathscr{Z} / \mathscr{X}}^{\vee}\right)-$ исключительньй дивизор раздутия $f$ и $g=f \mid \mathscr{D}$. Тогда из $[2,6.7]$ легко получаем изоморфизм градуированных групп

$$
A_{*}(\widetilde{\mathscr{X}}) \simeq f^{*} A_{*}(\mathscr{X}) \oplus j_{*} A_{*}(\mathscr{D} / \mathscr{Z}) \simeq A_{*}(\mathscr{X}) \oplus A_{*}(\mathscr{D} / \mathscr{Z}),
$$

где $j: \mathscr{D} \hookrightarrow \widetilde{\mathscr{X}}$ - вложение. В частности, при $r=1$ с учетом (11) имеем

$$
A_{*}(\widetilde{\mathscr{X}}) \simeq A_{*}(\mathscr{X}) \oplus g^{*} A_{*}(\mathscr{Z}) .
$$

Применим теперь эти замечания к теореме 1 . Из (10), (12) и (13) немедленно получаем изоморфизмы градуированных групп:

$$
\begin{gathered}
A_{*}\left(X_{1}\right) \simeq A_{*}(\widetilde{\Delta} / \Delta) \oplus A_{*}(H \times H), \quad A_{*}(\widetilde{X}) \simeq A_{*}\left(X_{1}\right) \oplus g_{E}^{*} A_{*}(\widetilde{\Gamma}), \\
A_{*}(D) \simeq g_{D}^{*} A_{*}(Y) \oplus A_{*}(Y), \quad A_{*}(\widetilde{X}) \simeq A_{*}(X) \oplus g_{D}^{*} A_{*}(Y) .
\end{gathered}
$$


Кроме того, утверждения (iii) и (v) теоремы 1 дают изоморфизмы градуированных групп:

$$
\begin{aligned}
A_{*}(D) & \simeq A_{*}(\widetilde{\Delta}) \oplus \gamma^{*} A_{*}(\widetilde{\Gamma} \cap \widetilde{\Delta}), \\
A_{*}(\widetilde{\Gamma} \cap \widetilde{\Delta}) & \simeq A_{*}(\widetilde{S \times S}) \oplus \delta^{*} A_{*}(\widetilde{S \times S}) .
\end{aligned}
$$

Применим теперь полученные изоморфизмы (14)-(16) к частному случаю $S=\mathbb{P}^{2}$. В этом случае легко видеть, что $\Delta \simeq H \simeq \mathbb{P}\left(S^{2} T_{\mathbb{P}^{2}}\right)$ и $\widetilde{S \times S} \simeq \mathbb{P}\left(T_{\mathbb{P}^{2}}\right) \times_{-\mathbb{P}^{2}} \mathbb{P}\left(T_{\mathbb{P}^{2}}\right)$. Отсюда немедленно имеем

$$
\operatorname{rank} A_{i}(\Delta)=\left\{\begin{array}{ll}
1, & i=0,4, \\
2, & i=1,3, \\
3, & i=2,
\end{array} \quad \operatorname{rank} A_{i}(\widetilde{S \times S})= \begin{cases}1, & i=0,4, \\
3, & i=1,3, \\
4, & i=2\end{cases}\right.
$$

Кроме того, если $\pi_{1}: \mathscr{V}=\mathbb{P}\left(T_{\mathbb{P}^{2}}(-1)\right) \rightarrow \mathbb{P}^{2}-$ структурньй морфизм проективизации и $\rho: \mathscr{V} \rightarrow \mathbb{P}^{2}$ - отображение линейньп рядом $\left|\mathscr{O}_{\mathscr{V} / \mathbb{P}^{2}}(1)\right|$, то нетрудно видеть, что $\widetilde{S \times S} \simeq \mathbb{P}\left(\rho^{*} T_{\mathbb{P}}^{2}\right)$ и естественная проекция $\widetilde{S \times S} \rightarrow S$ совпадает с композицией $\mathbb{P}\left(\rho^{*} T_{\mathbb{P}}^{2}\right) \stackrel{\pi_{2}}{\rightarrow} \mathscr{V} \stackrel{\pi_{1}}{\rightarrow} \mathbb{P}^{2}$, где $\pi_{2}-$ структурный морфизм. Отсюда и из утверждения (iіi) теоремы 1 , учитьвая (16), находим

$$
\operatorname{rank} A_{i}(\widetilde{\Gamma})=\left\{\begin{array}{ll}
1, & i=0,6, \\
6, & i=1,5, \\
14, & i=2,4, \\
18, & i=3,
\end{array} \quad \operatorname{rank} A_{i}(\widetilde{\Gamma} \cap \widetilde{\Delta})= \begin{cases}1, & i=0,5 \\
4, & i=1,4 \\
7, & i=2,3\end{cases}\right.
$$

Последовательная подстановка равенств (17), (18) в формулы $(14),(15)$ дает следующий результат.

Теорема 2. Ранги $a_{i}=\operatorname{rank} A_{i}(X), 0 \leqslant i \leqslant 8$, групn Чжоу многообразия $X$ полных пар двоеточий проективной плоскости $\mathbb{P}^{2}$ равны:

$$
a_{0}=a_{8}=1, \quad a_{1}=a_{7}=5, \quad a_{2}=a_{6}=16, \quad a_{3}=a_{5}=29, \quad a_{4}=36 .
$$

В случае $k=\mathbb{C}$ соответственно числа Бетти $b_{i}(X)$ и топологическая эйлерова характеристика $\chi_{\text {top }}(X)$ для $X=\mathbb{P}^{2}$ равны:

$$
b_{2 k}=a_{k}, \quad k=0, \ldots, 8, \quad b_{2 k+1}=0, \quad k \geqslant 0, \quad \chi_{\text {top }}(X)=138 .
$$

\section{СПИСОК ЦИТИРОВАННОЙ ЛИТЕРАТУРЫ}

[1] Тихомиров А. С. Многообразие полньх пар нульмерных подсхем алгебраической поверхности // Изв. РАН. Сер. матем. 1997. Т. 61. №6. С. 153-180.

[2] Фултон У. Теория пересечений. М.: Мир, 1989.

[3] Шафаревич И. Р. Основы алгебраической геометрии. Т. 1. М.: Наука, 1988.

[4] Мойшезон Б. Г. Об $n$-мерных компактных комплексных многообразиях, имеющих $n$ алгебраически независимых мероморфных функций. I // Изв. АН СССР. Сер. матем. 1966. Т. 30. №1. C. 133-174.

[5] Harris J. Algebraic Geometry. A First Course. New York: Springer, 1992.

Ярославский государственный педагогический университет им. К. Д. Ушинского 